\title{
La historia como recurso didáctico para la enseñanza de las matemáticas desde las directrices curriculares para la educación secundaria en Costa Rica (1949-2012)
}

\author{
Teaching Mathematics History as a Teaching Resource from High School Education \\ Curricular Guidelines in Costa Rica (1949-2012)
}

Leonel Castro-Soto

leonelm17@gmail.com

Universidad de Costa Rica,

San José, Costa Rica.

Patricia Cortés-Campos

patocc.1990@gmail.com

Universidad de Costa Rica,

San José, Costa Rica.

Roberto Guzmán-Gómez

robertoguzmang89@hotmail.com

Universidad de Costa Rica,

San José, Costa Rica.

Noemí Lezcano-Arias

noemilezcano@gmail.com

Universidad de Costa Rica,

San José, Costa Rica.

Grettel Mora-Coto

grettel19@hotmail.com

Universidad de Costa Rica,

San José, Costa Rica.

Natalia Rosales-Fernández

nataliarosafer@gmail.com

Universidad de Costa Rica,

San José, Costa Rica.

Miguel Picado-Alfaro

miguepicado@hotmail.com

Universidad de Costa Rica,

San José, Costa Rica.

Recibido-Received: 18/oct/2015 / Aceptado-Accepted: 1/dic/2015 / Publicado-Published: 31/jul /2016.

Leonel Castro-Soto, Patricia Cortés-Campos, Roberto Guzmán-Gómez, Noemí Lezcano-Arias, 
UNICIENCIA Vol. 30, No. 2, pp. 1-17. Julio-diciembre, 2016.

ISSN Electrónico: 2215-3470

URL: www.revistas.una.ac.cr/uniciencia

DOI: http://dx.doi.org/10.15359/ru.30-2.1

Email: revistauniciencia@una.cr

\begin{abstract}
Resumen
En Costa Rica, la reforma curricular de 2012 introdujo la historia de las matemáticas como un eje disciplinar otorgándole un papel protagónico en el proceso de enseñanza y aprendizaje. Este artículo describe la evolución que han tenido los programas de estudio como hilo conductor de la enseñanza de la matemática dentro de la educación secundaria en Costa Rica entre 1949 y 2012. Particularmente, destacan los planteamientos curriculares sobre el uso de la historia de la matemática como recurso didáctico. El estudio ha permitido reconocer dos tendencias específicas de este uso en los programas de estudio analizados: La historia es un elemento motivador para estudiantes, y una herramienta metodológica para la planificación didáctica del profesorado.
\end{abstract}

Palabras claves: Enseñanza secundaria, plan de estudios, investigación histórica, historia, aprendizaje.

\begin{abstract}
In Costa Rica, the 2012 curricular reform introduced the history of mathematics as a discipline central concept, awarding it a leading role in the teaching-learning process. This article describes the evolution that study programs have had as a thread for teaching mathematics in high school education in Costa Rica between 1949 and 2012. In particular, it highlights the curricular approaches on the use of the history of mathematics as a teaching resource. In the study programs analyzed, the study has allowed to recognize two specific trends of this use: History is a motivating element for students, and history is a didactic planning methodological tool for teaching staff.
\end{abstract}

Keywords: High school education, study plan, historic research, history, learning.

Desde diversos posicionamientos, distintos autores han reconocido la historia como un medio para comprender los orígenes y la evolución del conocimiento matemático. Esto, a partir de fenómenos o situaciones propias de los contextos social, cultural, político, económico y científico que han promovido este conocimiento (Babini y Pastor, 1997; Boyer, 2003; Collete, 1973; Fauvel y Gray, 1991).

La reforma curricular en matemáticas de 2012, promulgada por el Ministerio de Educación Pública de Costa Rica [MEP], otorga un papel protagónico a la historia de las matemáticas como uno de los ejes disciplinares para su enseñanza. Se reconocen en la historia distintos recursos valiosos para la acción de aula y su potencialidad para valorar las matemáticas como medio para el aprendizaje efectivo y la comprensión culta de las matemáticas.

Esta manera actual de acentuar la historia en la enseñanza y el aprendizaje de las matemáticas provoca una serie de cuestiones sobre su utilidad en el pasado. Esta se sintetiza en una cuestión general: ¿qué papel ha jugado la historia como recurso didáctico para la enseñanza de las matemáticas en Costa Rica antes de la Reforma Educativa en Matemáticas de 2012? Particularmente, ¿qué disposiciones curriculares han presentado los programas de estudio sobre el uso de la historia en la enseñanza de las matemáticas en periodos previos a esta norma?

El propósito del estudio que se presenta y, particularmente, el periodo temporal en que se ha delimitado, conducen a un análisis del contexto social, político y educativo en que se desarrollaron las acciones y fenómenos que caracterizan los procesos educativos antes, durante y después de la promulgación de la Constitución Política de 1949 y los acontecimientos previos a la Reforma curricular en matemática de 2012. Este artículo presenta un acercamiento a estos entornos y realidades como marco histórico para el análisis de los programas de estudio y el abordaje de la historia como eje disciplinar durante 1949 y 2012. 
Con especificidad, con el estudio se responde a dos objetivos particulares: (a) describir los contenidos sobre historia de la matemática presentes en la normativa curricular y los libros de texto de matemáticas para secundaria en Costa Rica en el periodo 1948-2012 y (b) caracterizar estos contenidos históricos mostrados en libros de texto de matemáticas de secundaria en el periodo 1948-2012.

\section{Antecedentes históricos}

Desde un marco general, la educación en Costa Rica tiene sus inicios en la Colonia (siglos XVI-XVII), etapa caracterizada por la influencia de los fines eclesiásticos y los intereses de las clases sociales acaudaladas en los procesos educativos. Es decir, en aquella época donde existía una marcada diferencia entre la educación para hombres y para mujeres, donde solo las familias con una economía estable podían enviar a sus descendientes a un centro educativo. El acceso a la educación en los distintos niveles era limitado.

A partir de 1940 se reconocen situaciones sociopolíticas que inciden en modificaciones importantes en las condiciones del sistema educativo costarricense (Castro, et al., 2015). Estas particularidades sustentaron múltiples decisiones que incidieron, de manera directa, en las propuestas curriculares para la enseñanza de las matemáticas y de otras disciplinas.

Particularmente, para educación primaria el presupuesto destinado era estable. Esto condujo a un aumento en la población matriculada en este nivel educativo entre 1890 y 1940 (Quesada, 2003). El énfasis era asegurar la primera enseñanza, una enseñanza básica para la población.

Por su parte, en educación secundaria la situación era distinta. Ruiz y Barrantes (2000) destacan que "la población que tenía acceso a este nivel de instrucción era sumamente restringida, en 1885 solamente funcionaban en el país cuatro centros de segunda enseñanza" (p. 146). Para inicios del siglo XX las instituciones para la educación secundaria se ubicaban en las provincias centrales del país. El Colegio San Luis Gonzaga, fundado en 1867, en la provincia de Cartago, la primera capital del país; el Liceo de Costa Rica y el Colegio Superior de Señoritas -fundados en 1887 y 1888 respectivamente- se localizaban en la provincia de San José; el Instituto de Alajuela, creado en 1887; y el Liceo de Heredia, fundado en 1887.

En cuanto a la educación superior, a partir de 1920 se inician esfuerzos para la creación de centros de estudios universitarios fundamentalmente en el centro del país.

Los cambios en la educación costarricense, en especial en primaria, en cuanto a cobertura, acceso y reformas curriculares, constituyeron las bases para un mayor desarrollo a partir de la década de los años 40. La ampliación de la cobertura educativa nacional, mediante la creación de nuevos centros de estudio, constituyó uno de los principales aciertos. La democratización de la enseñanza media, el inicio de la educación técnica y la expansión progresiva de la instrucción primaria son evidencias históricas de este proceso de expansión. También, es destacable en este periodo la propagación de la enseñanza secundaria: a finales de 1946 las siete provincias del país contaban con colegios oficiales (Dengo, 1995).

Desde 1940 hasta la década de los años 70, inclusive, el país experimentó una serie de reformas políticas que incidieron en cambios al sistema educativo. En la educación superior, destaca la creación de la Universidad de Costa Rica (UCR) en 1940, institución que comenzaría a encargarse de la formación del profesorado de educación secundaria mediante sus facultades de Ciencias y de Filosofía y Letras (Dengo, 2001). 
UNICIENCIA Vol. 30, No. 2, pp. 1-17. Julio-diciembre, 2016.

ISSN Electrónico: 2215-3470

URL: www.revistas.una.ac.cr/uniciencia

Email: revistauniciencia@una.cr

\section{La Constitución Política de 1949 y sus repercusiones en la educación}

En 1948 ocurre "un fenómeno social sin precedentes en la historia de nuestro país, la Guerra Civil del '48', génesis de la abolición del ejército, la fundación de la Segunda República, situaciones que derivan la promulgación de la Constitución de 1949" (Martínez, 2012, p. 24).

La Constitución Política de 1949 establece "la gratuidad de la enseñanza media... [e] introduce, por primera vez en el país, una articulación integral del sistema escolar, siguiendo la concepción de escuela unificada" ( Retana, 2010, p. 11). Con esta articulación del sistema educativo se pretendía una educación integral que involucrara y promoviera una coherencia curricular desde la educación preescolar hasta la formación universitaria.

Para los años 50 el país entra en un proceso de expansión económica que tuvo sus repercusiones en el sistema educativo, beneficiando la cobertura educativa y correspondiendo a los dictámenes de la Constitución de 1949 para asegurar la gratuidad de la educación y, con ello, la atención al creciente número de estudiantes en los diferentes sectores educativos.

En 1957 se promulgó la Ley Fundamental de Educación que establecía diversas regulaciones a los fines generales de la instrucción pública, con detalle a la educación preescolar, la educación primaria y la educación secundaria.

Durante la década de 1960 se acentúan una serie de avances en el sector educativo, producto de la estabilidad política y económica del país (Martínez, 2012). Para 1970 se percibe un estancamiento en el desarrollo de la educación primaria y la educación secundaria; la crisis económica que vivía el país tuvo sus repercusiones en el sistema educativo, derivando el cese de personal docente y la reducción del presupuesto para este sector. Los avances en esta década se vinculan con la educación superior. Se crean, en 1971 el Instituto Tecnológico de Costa Rica (ITCR) con sede central en Cartago, en 1973 la Universidad Nacional de Costa Rica (UNA) con sede en Heredia y para 1977 la Universidad Estatal a Distancia (UNED). En 1973 se establece el Plan Nacional de Desarrollo Educativo (PNDE) para sentar los precedentes de un proceso de modernización al sector educativo en sus diferentes niveles durante, al menos, una década (Dengo, 1995).

En la década de los años 80 se presenta una crisis que afecta la educación costarricense. Rama (1994) plantea que esta crisis "afecta a educadores y a la calidad de la educación; se reducen los recursos financieros lo cual - sumado a un conjunto de procesos sociales y otros, específicamente educativos - afecta cuantitativamente y cualitativamente la totalidad del sistema educativo" (p. 85). Para contrarrestar estas incidencias se establecen diferentes propuestas económicas como los Programas de Ajuste Estructural (PAE) que tienen como consecuencia el fortalecimiento del sector privado de la educación. Por otra parte, uno de los sectores que más beneficio obtuvo fue el de la educación superior, principalmente en el ámbito privado, a través de la expansión de las universidades y las instituciones parauniversitarias privadas.

A partir de 1990, los cambios que se efectúan en la educación se vinculan directamente con la organización curricular: modificaciones al currículo basadas en diferentes postulados de las políticas nacionales e internacionales, como la Declaración Universal de la Educación para Todos (UNESCO, 1990) y la Política Educativa hacia el Siglo XXI. Aunado a esto, se aprueba una política curricular como fundamento para la aprobación de los programas de estudio que en lo sucesivo se ejecutarían. "Estos programas fueron modificados y aplicados consecutivamente desde 1991 hasta 1993 para los diversos niveles y especialidades” (Martínez, 2012, p. 32). 
En los primeros años del siglo XXI los cambios que comenzaron a gestarse en el ámbito educativo tuvieron como objetivo el posicionamiento curricular, es decir, centraron su atención en los enfoques, metodología y estructura organizacional de los programas de estudio considerados.

Recientemente, se implementaron nuevas reformas curriculares para la educación musical, educación física, educación cívica, ciencias, español y matemáticas. Esta última fue aprobada en 2012 y tuvo como objetivo la modificación de la malla curricular desde el primer ciclo de la educación general básica (EGB) hasta la educación diversificada (Castro, et al., 2015).

A manera de síntesis, el sistema educativo costarricense tuvo un cambio importante con la promulgación de la Constitución Política de 1949. A partir de ese momento el ámbito educativo entra en un periodo de reformas influenciadas por políticas nacionales e internacionales que le han dado fundamento y lo han fortalecido.

\section{Etapas históricas}

Los acontecimientos descritos permiten reconocer momentos "cumbre" en el contexto político-educativo costarricense durante 1949-2012. Siguiendo a Picado (2009), se ha acotado este periodo en cuatro etapas históricas, de interés tanto político como educativo.

\section{Etapa I: Etapa de promulgación constitucional (1949 - 1963)}

Toma como punto de partida los cambios a nivel político, social y económico, basados en la Constitución de 1949, que condujeron a una cantidad importante de reformas al sistema educativo costarricense. Estas reformas derivaron en la amplitud de la cobertura educativa y la obligatoriedad de la educación. Promovieron la creación de nuevos centros de estudio principalmente en la enseñanza en educación secundaria y en preescolar. En matemática, en esta primera etapa sobresalen los preliminares de la reforma hacia las matemáticas modernas, que a partir de 1964 traería diferentes cambios curriculares en la asignatura.

\section{Etapa II: Etapa de implementación de las matemáticas modernas (1964 - 1979)}

A partir de 1964, como consecuencia de la reforma de las matemáticas modernas, inicia la implementación de programas de estudio con una marcada influencia reformista. Estos programas fueron aplicados de manera sucesiva hasta 1968 y otorgaron a las matemáticas una mayor presencia en la educación que la brindada hasta el momento. Posteriormente, se proclaman y ejecutan los programas de estudio para esta asignatura en los años 1972 y 1979, los cuales seguían aún bajo la influencia de esta reforma.

\section{Etapa III: Etapa de conceptualización curricular de los procesos de enseñanza y aprendizaje (1980 - 1994)}

En esta etapa, el país entra en una crisis provocada por los cambios económicos que afectó a sectores como la educación y la salud. Se comienzan a aplicar los programas de ajuste estructural como mecanismo de confrontación a esta crisis. También, inicia la expansión de la educación superior privada. Por otra parte, se mantiene una constancia respecto a los cambios de índole curricular; no es sino hasta 1991 cuando se realiza una nueva propuesta curricular 
que contempla cambios vinculados a contenidos y enfoques curriculares. En 1990 se lleva a cabo la Declaración Mundial sobre Educación para Todos, en la cual se insta a las diferentes sociedades a velar por el acceso igualitario a la educación de su ciudadanía. Esto obliga al sistema educativo a la reestructuración de las políticas educativas con miras a satisfacer las necesidades de toda la población.

\section{Etapa IV: Etapa de implementación y reajustes a la conceptualización curricular de los} procesos de enseñanza y aprendizaje (1995 - 2012)

Durante esta etapa se continuaron los cambios tanto a nivel macro y micro de la educación en Costa Rica, con la implementación de reformas curriculares que han intentado alcanzar las propuestas curriculares nacionales e internacionales. Se plantea la Política Educativa hacia el Siglo XXI para fomentar el desarrollo educativo integral. En cuanto a cambios curriculares, en esta etapa, se presentan reformas a los programas a nivel general, que incluyen el paso del aprendizaje conductual a la introducción de las propuestas que apoyan el aprendizaje de forma constructivista. Por último, la etapa culmina con el cambio curricular de manera general que se plantea con el nuevo programa de estudio para matemáticas aprobado en el año 2012. Este dispone la reestructuración global de cada uno de los programas de estudio de primaria y educación secundaria para articular, de mejor manera, los diferentes conocimientos matemáticos que debe adquirir el estudiantado para un mejor desarrollo social, personal y profesional y enfrentarse a los retos que le imponga la sociedad.

\section{Marco teórico}

El estudio se fundamenta en diversas investigaciones desarrolladas sobre historia de la educación matemática que destacan particularidades de los sistemas educativos en la formación de estudiantes y maestros, y sus implicaciones en la elaboración de textos educativos (Carrillo, 2005; López, 2011; Maz, 2005; Picado, 2012).

También, toma como marco de referencia las políticas nacionales que se han derivado de diferentes tendencias internacionales y que han provocado cambios en la educación costarricense, particularmente en la enseñanza de la matemática en educación secundaria.

El estudio de las diferentes normativas curriculares permite realizar una descripción de estas y reconocer las especificidades del contexto político-educativo-social de la época en que se delimita la investigación. El interés radica en identificar aquellos aspectos vinculados con la historia de la matemática, contemplados en los programas de estudio, procura analizar el uso (o usos) de la historia de la matemática en los procesos de enseñanza y aprendizaje en Costa Rica.

Sobre los usos de la historia en las matemáticas, Sierra (1997)destaca una serie de actividades que pueden implementarse en la enseñanza. Entre estas, la presentación de pasajes de la historia, la introducción y presentación de problemas, el análisis de sus respectivas soluciones históricas, la reproducción del proceso de aprendizaje de un área particular, la lectura de documentos originales de reconocidos matemáticos en la producción de conocimiento matemático; todo lo cual incentiva el aprendizaje escolar. 
ISSN Electrónico: 2215-3470

DOI: http://dx.doi.org/10.15359/ru.30-2.1
UNICIENCIA Vol. 30, No. 2, pp. 1-17. Julio-diciembre, 2016. URL: www.revistas.una.ac.cr/uniciencia Email: revistauniciencia@una.cr

Aunado a esto, algunas actividades potencian la diversidad y riqueza de la planificación del profesorado y son, además, recursos pedagógicos para el uso de la historia de las matemáticas en su enseñanza. Por ejemplo, impartir clases de historia de las matemáticas, elaborar materiales y proyectos con un tema histórico, explorar los errores del pasado para ilustrar técnicas y métodos de resolución, idear aproximaciones pedagógicas al tema según su desarrollo histórico y realizar proyectos relacionados con la historia de la actividad matemática local.

Desde estas perspectivas, se enfoca el análisis de programas de estudio y libros de texto para reconocer las peculiaridades de estos usos en la normativa curricular costarricense desde 1949 a 2012. Se entiende el libro de texto como "una publicación especializada, con identidad propia, que nace en respuesta a las necesidades del sistema general y público de enseñanza y del modelo de enseñanza simultánea" (Gómez, 2000, p. 77). Así, resaltan las implicaciones de los programas de estudio en los libros de texto, a partir de las indicaciones legales para el uso de la historia en la enseñanza y el aprendizaje de las matemáticas en nuestro país.

\section{Metodología}

El estudio se enmarca dentro de las investigaciones cualitativas-descriptivas basadas en el análisis de textos históricos como fuentes primarias de investigación; así como el estudio de los directrices curriculares vinculadas al recurso histórico desde los programas de estudio para educación secundaria.

\section{Selección de las fuentes}

Las fuentes de información son los Programas de Estudio de Matemática para Educación Secundaria en Costa Rica, entre 1949 y 2012. Estos se han localizado en bibliotecas y en los archivos personales de docentes de matemática. Los documentos seleccionados son:

- Programa de matemáticas de la Secretaría de Instrucción Pública durante 1904-1929: Educación secundaria

- Programa de matemáticas de 1939

- Programa de matemáticas de 1951

- Programa de matemáticas de 1964

- Programa de estudios de 1972: Tercer ciclo

- Programa de matemáticas de 1972 y 1979: Cuarto año

- Programa de estudios de 1979: III ciclo

- Programa de estudios de 1979: Educación diversificada quinto año y optativo

- Programa de estudios de 1987: Tercer ciclo y educación diversificada

- Programa de estudios de 1995: Tercer ciclo y educación diversificada

- Educación diversificada matemática de 1996

- Programa de estudios de 2000: Matemática III ciclo

- Programa de estudios de 2001: Matemática para la educación diversificada

- Programas de estudios educación diversificada de 2005

- Programas de estudios de 2012: III ciclo de educación general básica y educación diversificada 


\section{Análisis de las fuentes}

El análisis de las fuentes ha considerado la revisión de los programas de estudio, resaltando aquellos aspectos relacionados con la historia de la matemática, como lineamientos generales y específicos aplicables en la enseñanza-aprendizaje de conceptos matemáticos.

A continuación, se describen brevemente los programas de estudio de matemática para educación secundaria seleccionados, destacando la presencia de disposiciones sobre el uso de la historia de la matemática como recurso metodológico en los procesos de enseñanza-aprendizaje y eje disciplinar en el currículo.

\section{Programa de matemáticas de la Secretaría de Instrucción Pública durante 1904-1929: Educación secundaria}

Este programa de estudio refleja las intenciones educativas dirigidas al aprendizaje memorístico, que supone la aplicación de los conceptos matemáticos transmitidos al alumnado, donde "la austeridad y el rigor de la lógica matemática excluyen de sus enseñanzas las divagaciones oratorias... El cálculo perfeccionado y sabiamente aplicado se ha convertido en el instrumento más activo de nuestros conocimientos" (p. 1).

La enseñanza de las matemáticas en los liceos tiene por objeto principal, cultivar el tino lógico y obtener seguridad y habilidad en el cálculo, tanto en números ordinarios como en algebraicos; desarrollar la intuición geométrica y obtener práctica y destreza en el dibujo de figuras. Los alumnos se ejercitarán en el lenguaje correcto. (p. 1)

El método de enseñanza tradicionalista toma fuerza, formando al estudiantado hacia la aplicación de métodos matemáticos idóneos y la implementación de un lenguaje acorde con los problemas planteados por cada docente.

\section{Programa de matemáticas de 1939}

Este programa de estudio conlleva a la aplicación de procesos matemáticos formales, como la demostración de teoremas o propiedades matemáticas, dirigiendo a los estudiantes a la aplicación de estos resultados en ejercicios propuestos por el personal docente. Según estos planteamientos se pretendía:

Despertar en los alumnos el verdadero espíritu matemático que se adquiere, en forma especial, por medio de la resolución de problemas de carácter práctico en los cuales han de aplicar teoremas y fórmulas cuya existencia deben conocer sin dar, eso sí, importancia fundamental de la demostración de esos teoremas y a la investigación de esas fórmulas. (p. 31)

\section{Programa de matemáticas de 1951}

El programa de estudio incentiva la adquisición de conocimientos por parte del alumnado, precisando en su lenguaje -de estudiantes- al resolver determinado problema matemático, con muestras de seguridad y precisión en su solución. Se redirige la práctica educativa hacia procesos mecánicos, sin dejar de lado la reflexión y la búsqueda de logros adquiridos a través de la resolución de problemas. "Nuestra enseñanza debe presentar a los jóvenes ocasiones en que 
ellos puedan gozar de la fina alegría del éxito, y de oportunidades para manifestar y aprovechar su fantasía, para fortalecer su tenacidad, su perseverancia” (p. 39).

De esta manera, la enseñanza de la matemática logrará incentivar motivación en los estudiantes hacia la disciplina, creando espacios para la investigación y la aplicación de los conocimientos adquiridos, mediante una conexión inmediata con los diferentes contextos y la interdisciplinariedad. Se eliminaron problemas difíciles, abordando los conocimientos indispensables para el desarrollo de la materia y la comprensión de temas más avanzados; al impartir el conocimiento se describía solo un camino, el más sencillo y los ejercicios se proponían de acuerdo con el desarrollo mental con materiales ilustrativos: construcciones geométricas y construcción de sólidos, hábitos de medición y representación gráfica.

\section{Programa de matemáticas de 1964}

Los objetivos establecidos en este programa otorgan mayor protagonismo al alumnado, al desarrollar o potenciar la habilidad de resolver problemas matemáticos que propicien el razonamiento, el cálculo y la utilización del método científico, con lo cual se fomentan los hábitos del precisión, orden y claridad en el uso de principios y leyes de la matemática (Castro el al., 2015). Se pretende, de esta manera, la relación entre las matemáticas y los procesos culturales y sociales, enfatizando en la importancia de las aplicaciones reales.

En relación con los contenidos abordados y su vínculo con la historia de la matemática, se establece la necesidad de incluir subtemas que incorporan la historia de la matemática, como por ejemplo: bosquejo histórico sobre el desarrollo de las matemáticas; reseña histórica sobre los numerales (sistemas posicionales y no posicionales con diferentes bases; los numerales babilonios, los egipcios, los mayas y los romanos); en el sexto tema "Números Racionales" (p. 54), se vuelve a involucrar la historia de la matemática al destacar la historia de las fracciones.

\section{Programa de estudios de 1972: Tercer ciclo}

Como finalidades en este programa de estudios se expone la comprensión y formulación de la matemática como un sistema interrelacionado, específicamente en la enseñanza de conceptos matemáticos a través del desarrollo histórico de la matemática.

Los contenidos para el primer año incluyen, en la introducción, una definición de matemática y un bosquejo histórico sobre su desarrollo. También, se abordan sus aplicaciones, destacando los sistemas de numeración usados en la antigüedad.

Sugieren la implementación de actividades como el periódico mural, la presentación de biografías de matemáticos y artículos referentes al tema.

Para el abordaje de los números racionales se recomienda un resumen de carácter histórico sobre su desarrollo; en álgebra y geometría se sugiere un periódico mural con biografías de matemáticos que hayan contribuido a su desarrollo.

\section{Programa de Matemáticas de 1972 y 1979: Cuarto año}

Se establece la correspondencia entre el recurso histórico y los contenidos abordados en el programa. "Las Matemáticas constituyen una ciencia viva, llena de dinamismo y no solamente un producto inerte y rígido heredado de la antigüedad" (p. 1). 
En cuanto al uso de la historia en las lecciones de matemática, se plantea la incorporación de menciones históricas que ilustren el acontecer y evolución de las matemáticas. Por ejemplo, se puede optar por abordar el tema de logaritmos. Se esbozan los contenidos relacionados con el curso optativo sobre estructura algebraica, matrices y probabilidades, justificado a partir de la relación con otras disciplinas como física y química y su aplicabilidad en las actividades de la vida cotidiana.

\section{Programa de Estudios de 1979: III ciclo}

Como orientaciones de enseñanza, se plantea el desarrollo de estrategias metodológicas diversas como investigaciones y resúmenes relacionados con acontecimientos históricos acerca del origen de algunos conceptos matemáticos, así como aspectos trascendentales de personajes matemáticos. La inclusión de contenidos históricos en las lecciones de matemática debe ser abordada durante el III ciclo de la EGB.

Estas actividades deben establecer una correspondencia con respecto a los contenidos presentados desde el proceso de enseñanza-aprendizaje y la historia de la matemática como recurso mediador. Sin embargo, no se muestra con claridad el objetivo pretendido al utilizar la historia dentro del programa de estudio.

\section{Programa de estudios de 1979: Educación diversificada quinto año y optativo}

Este programa de estudios plantea una serie de objetivos generales para el ciclo escolar y los objetivos para la educación matemática del ciclo diversificado, sin puntualizar el uso de la historia de la matemática en el proceso de enseñanza y aprendizaje.

Como finalidades de enseñanza para contenidos específicos, vinculadas a la historia de la matemática, se plantean:

- Comprender que el conjunto de los reales es un subconjunto propio de los complejos

- Investigar los orígenes remotos de la geometría.

- Comprender los orígenes históricos de cálculo infinitesimal y su problemática. Aunado a esto, se contemplan actividades como la elaboración de esquemas, comentarios e investigaciones sobre el desarrollo histórico del cálculo infinitesimal.

No obstante, pese a que se identifican objetivos de enseñanza con vínculo históricos, no se reconocen sugerencias sobre cómo incorporar dicho recurso en las lecciones de matemática.

\section{Programa de estudios de 1987: Tercer ciclo y educación diversificada}

En este programa no se hace mención explícita de la utilización de aspectos históricos para desarrollar los diferentes procesos de enseñanza y aprendizaje de la matemática, tampoco se evidencia la presencia de contenidos que puedan ser vinculados con la historia de la matemática.

No obstante, en 1986, para el nivel de sexto año de la educación primaria, se consideraba el uso de la historia en los contenidos expuestos en el programa. En la serie de libros Hacia el Siglo XXI, se contemplan diferentes aspectos históricos. Por ejemplo, en el libro para sexto año se describe que el "sistema de numeración decimal fue inventado por los hindúes y llevado a toda Europa a través de sus transacciones comerciales por los árabes. Más tarde se difundió por todo el mundo” (MEP, 1986, p.15). 
ISSN Electrónico: 2215-3470

DOI: http://dx.doi.org/10.15359/ru.30-2.1
UNICIENCIA Vol. 30, No. 2, pp. 1-17. Julio-diciembre, 2016. URL: www.revistas.una.ac.cr/uniciencia Email: revistauniciencia@una.cr

\section{Programa de estudios de 1995: tercer ciclo y educación diversificada}

En el programa se presenta la enseñanza de la matemática como un proceso continuo y conjunto en el cual es trascendental "valorar las contribuciones de los antiguos pensadores en el desarrollo de la Matemática” (p. 3). Específicamente en este documento, la historia de la matemática se incluye dentro los diversos contenidos.

- En geometría se propone la obtención, selección y análisis de información que justifica el nacimiento y desarrollo de la geometría.

- En aritmética se toma conciencia de la necesidad de los números enteros y racionales, como parte del proceso evolutivo en el desarrollo del conocimiento matemático.

- En álgebra se espera que, por medio de la historia, se investigue la utilidad de esta rama de la disciplina en otros campos del conocimiento.

- Para trigonometría se procura reconocer los aportes de la matemática como instrumento para el desarrollo de otras disciplinas.

\section{Educación diversificada matemática de 1996}

Este programa de estudio responde a la política educativa Hacia el siglo XXI. Se manifiesta la importancia de visualizar la matemática como fuente importante para la transmisión de valores y actitudes en el alumnado. Su estudio contribuye "a la formación de valores morales y éticos, a perfeccionar el uso del idioma, a valorar las contribuciones de los antiguos pensadores en el desarrollo de la Matemática” (p. 1).

En el marco de las orientaciones metodológicas, se concibe la enseñanza de la matemática cual herramienta que implementa la resolución de problemas como estrategia para la enseñanza y aprendizaje de conceptos matemáticos. Además, la evolución y desarrollo de algunos conceptos predisponen a la realidad histórica, importancia y contribución de las matemáticas al desarrollo de la humanidad, lo cual funge como un factor motivador en la formación de estudiantes.

Para el ciclo diversificado se dividen los contenidos: geometría, álgebra, análisis y trigonometría. Estas áreas contemplan temas predispuestos y orientaciones para la evaluación de los aprendizajes.

Como objetivos vinculados con la historia de la matemática en la educación diversificada se proponen:

- Promover la investigación sobre los aportes de la matemática en los avances científicos y tecnológicos que han contribuido al progreso y bienestar del individuo en la sociedad.

- Relacionar la matemática con la realidad inmediata como disciplina ampliamente vinculada al quehacer humano para lograr una persona competente en el campo en el que se desenvuelva.

- En geometría y álgebra para $10^{\circ}$ año se contemplan finalidades como:

- Valorar los principales acontecimientos en el desarrollo histórico de la geometría y sus implicaciones en el desarrollo científico y tecnológico.

- Valorar los principales aportes del álgebra al desarrollo científico y tecnológico, mediante el análisis de hechos históricos. 
En estas áreas se enfatiza en diferentes aspectos relacionados con la historia de la matemática. En geometría se propone la "obtención, selección y análisis de información que justifique el aporte histórico de la intervención de la rueda en el desarrollo tecnológico” (p. 11). Además, como criterio de evaluación relacionado al mismo concepto, se enfatiza en interpretar y describir algunos "hechos relacionados con la invención de la rueda, que justifiquen la repercusión del desarrollo de la geometría en la calidad de vida” (p. 12). En álgebra, de manera similar, en los procedimientos se establece la "obtención, selección, y análisis de información relativa al desarrollo histórico del álgebra y sus implicaciones en la ciencia y tecnología" (p. 13). Con los valores y actitudes deseables en estudiantes se busca fomentar la interpretación de "hechos relevantes en la historia del álgebra que inciden en el desarrollo de la matemática y sus repercusiones en la ciencia y la tecnología" (p. 14).

Para $11^{\circ}$ año, los contenidos propuestos incluyen funciones, límites y sus propiedades, límites y sus derivados, y trigonometría. Como valores y actitudes deseables se contemplan en las áreas de límites y sus propiedades, la toma de conciencia "del proceso evolutivo en el desarrollo del conocimiento matemático" (p. 20).

\section{Programa de estudios de 2000: Matemática III Ciclo}

El programa plantea involucrar al estudiantado en el devenir histórico de la matemática; esto contribuye a que conozca la realidad histórica y evolutiva del conocimiento matemático, despertando su motivación e interés hacia la disciplina. En este programa de estudio se describen los siguientes objetivos generales relacionados con la historia de la matemática:

- Promover la investigación sobre los aportes de la matemática al desarrollo de la cultura.

- Promover la investigación sobre los aportes de la aritmética, álgebra, geometría, trigonometría y estadística, en los avances científicos y tecnológicos que se han logrado a través de la historia, que a la vez han contribuido al progreso y bienestar del individuo y de la sociedad.

\section{Programa de estudios de 2001: Matemática para la educación diversificada}

Como aspecto por considerar se supone la utilización de elementos históricos para enseñar ciertos conocimientos matemáticos. La enseñanza de la matemática "contribuye con la formación de valores morales y éticos, a perfeccionar el uso del idioma, a valorar las contribuciones de los antiguos pensadores en el desarrollo de la Matemática" (p. 10). Se pretende, además, que el alumnado obtenga experiencias de aprendizaje relacionadas con "la evolución histórica, cultural y científica de las matemáticas, de forma que puedan apreciar el papel que cumplen las matemáticas en el desarrollo de nuestra sociedad y el impacto que tienen en la cultura y la vida diaria” (p. 11).

Como objetivos generales vinculados con aspectos históricos se plantea:

- Incorporar datos históricos al "promover la investigación acerca de los aportes de la Matemática en los avances científicos y tecnológicos que han contribuido al progreso y bienestar del individuo en la sociedad" (p. 42).

- En geometría, se pretende el "análisis de información que justifique el aporte histórico de la invención de la rueda, en el desarrollo tecnológico” (p. 73) 
ISSN Electrónico: 2215-3470

DOI: http://dx.doi.org/10.15359/ru.30-2.1
UNICIENCIA Vol. 30, No. 2, pp. 1-17. Julio-diciembre, 2016. URL: www.revistas.una.ac.cr/uniciencia Email: revistauniciencia@una.cr

- Para trigonometría se recomienda la "utilización de diferentes estrategias que permitan exteriorizar juicios de valor para apreciar la importancia de la trigonometría a través de la historia de la humanidad" (p. 85).

\section{Programas de estudios educación diversificada de 2005}

Este programa conserva el planteamiento del programa de estudios para III Ciclo de la EGB. Con su ejecución se pretende que el estudiantado adquiera "experiencias variadas en relación con la evolución cultural, histórica y científica de las matemáticas, de forma que puedan apreciar el papel que cumplen las matemáticas en el desarrollo de nuestra sociedad" (p. 14).

\section{Programas de estudios de 2012: III ciclo de educación general básica y educación diversificada}

Laúltima reforma los programas de estudios de matemática se da en el 2012. Esta reestructuración supone diversos y variados aspectos para el desarrollo de las lecciones de matemática, como la implementación de estrategias metodológicas y el reajuste en cuanto a la cantidad de contenidos por enseñar en cada ciclo lectivo, desde educación primaria hasta educación secundaria.

Uno de los pilares fundamentales en estos programas de estudio es la incorporación del recurso histórico en el desarrollo de contenidos, o como complemento de estos. Sobre ello:

La Historia de la Matemática permite romper con el esquema de que las Matemáticas son una colección de axiomas, teoremas, pruebas y donde lo esencial es la claridad lógica de sus argumentos. Al colocar los objetos matemáticos en contextos socioculturales se permite visualizar la participación de heurísticas, dudas, errores, concepciones equivocadas e incluso la existencia de retrocesos cognoscitivos en algunos campos. (MEP, 2012, p. 39)

Los planteamientos curriculares consideran la historia de la matemática como un recurso mediador de enseñanza que favorece una visión humanista de la asignatura, pues conlleva a una construcción sociocultural. También la utilización de la historia propicia recursos de aula, y fomenta una valoración y comprensión culta de la matemática, valiosa para la formación de la ciudadanía. La formulación de actividades didácticas y metodológicas vinculadas a la historia de la matemática sugiere:

- En aritmética, implementar reseñas históricas que ilustren el nacimiento de los números irracionales como medio de resolución de algunos problemas en los cuales los números racionales eran insuficientes.

- En geometría, optar por la introducción del tema de semejanza de triángulos a través de una breve reseña biográfica sobre Tales de Mileto.

- Para la unidad de relaciones y álgebra, acentuar la relación existente del número áureo con la historia, el arte, la filosofía y otras disciplinas.

\section{Resultados}

Los programas de estudio analizados surgen como respuesta a las diferentes reformas educativas que se incentivaron para el fortalecimiento del sistema educativo en distintos momentos de la historia social y política de Costa Rica, particularmente en la educación matemática. La tabla 1 resume los objetivos vinculados a la historia de la matemática, contemplados en los programas de estudio de cada etapa histórica, con el fin de mostrar su evolución a lo largo del periodo de 1949-2012. 
UNICIENCIA Vol. 30, No. 2, pp. 1-17. Julio-diciembre, 2016.

ISSN Electrónico: 2215-3470

URL: www.revistas.una.ac.cr/uniciencia

DOI: http://dx.doi.org/10.15359/ru.30-2.1

Email: revistauniciencia@una.cr

Tabla 1

Objetivos de los programas estudio por etapas históricas

\begin{tabular}{lr}
\multicolumn{1}{c}{ Programas de estudio } & Aspectos relacionados con la historia de la matemática \\
\hline - $\begin{array}{l}\text { Programa de matemáticas de la Secretaría } \\
\text { de Instrucción Pública durante 1904- }\end{array}$ & $\begin{array}{l}\text { No se presentan aspectos vinculados con la historia de la } \\
\text { matemática y la enseñanza de conceptos matemáticos. }\end{array}$ \\
- $\begin{array}{l}\text { 1929: Educación secundaria. } \\
\text { - Programa de matemáticas de 1939 }\end{array}$ & \\
\hline
\end{tabular}

Etapa II: 1964 - 1979

- Programa de matemáticas de 1964.

- Estrategias metodológicas como elaboración de

- Programa de estudios de 1972: tercer ciclo

- Programa de matemáticas de 1972 y 1979 : bosquejos históricos sobre el desarrollo de las cuarto año. matemáticas, reseñas históricas de conceptos, periódico mural y biografía de la matemática,

- Programa de estudios: III ciclo, MEP 1979. biografía de matemáticos y artículos referentes al tema, incorporación de menciones históricas que ilustren el acontecer y evolución de las matemáticas.

- Programa de estudios de 1979: Educación diversificada quinto año y optativo

- Abordaje de los temas a partir de investigaciones y resúmenes relacionados con acontecimientos históricos acerca del origen de algunos conceptos matemáticos.

- Todos los aspectos resaltados se encuentran relacionados con conceptos matemáticos en las áreas de aritmética, álgebra y geometría.

Etapa III: 1980 - 1994

- Programa de estudios. Tercer ciclo y educación diversificada, 1987.

- No se sugiere la utilización de aspectos históricos dentro de los procesos de enseñanza y aprendizaje.

Etapa IV: 1995 - 2012

- Programa de estudios: Tercer ciclo MEP y

- Se destaca la importancia de la matemática para el educación diversificada, 1995.

- Educación diversificada. Matemática, 1996.

- Programa de estudios: Matemática III ciclo, 2000.

- Programa de estudios 2001: Matemática para la educación diversificada.

- Programas de estudios. Educación diversificada, 2005

- Programas de estudios: III ciclo de educación general básica y educación diversificada, 2012. desarrollo de la humanidad, al valorar las contribuciones de los antiguos pensadores en el desarrollo de la matemática.

- Se incentiva la investigación sobre la utilidad de esta disciplina con otros campos del conocimiento y las experiencias de aprendizaje relacionadas con la evolución histórica, cultural y científica de las matemáticas, de forma que pueda apreciarse el papel que cumplen las matemáticas en el desarrollo de nuestra sociedad y el impacto que tienen en la cultura y la vida diaria.

- Se promueve una visión humanista de la disciplina en medio de una construcción sociocultural.

- Estos aspectos vinculados con la historia de la matemática se establecen en las áreas de aritmética, álgebra, geometría y trigonometría.

Nota: MEP = Ministerio de Educación Pública. Elaboración propia. 
ISSN Electrónico: 2215-3470

DOI: http://dx.doi.org/10.15359/ru.30-2.1
UNICIENCIA Vol. 30, No. 2, pp. 1-17. Julio-diciembre, 2016. URL: www.revistas.una.ac.cr/uniciencia Email: revistauniciencia@una.cr

Los programas de estudio a partir de 1964 pretendían resaltar el vínculo entre el origen y evolución de los conocimientos matemáticos, situación que en la I etapa no se contemplaba como finalidad de enseñanza.

A partir de la etapa de implementación de las matemáticas modernas (1964 - 1979), los programas de estudio presentan aspectos históricos de la matemática en los procesos de enseñanza y aprendizaje de conceptos matemáticos, principalmente en álgebra, aritmética y geometría. Esta incorporación presenta un crecimiento continuo en sugerencias metodológicas a lo largo del periodo; todo ello fruto de múltiples reformas que sugieren la aplicación de estrategias y metodológicas novedosas en las lecciones de matemática y de las acciones didácticas a nivel internacional sobre el uso de la historia en educación matemática que repercuten en estos cambios curriculares.

Los programas de estudio consideran la historia de la matemática como recurso metodológico que permite mostrar la evolución de esta disciplina, a partir del aporte de civilizaciones y personajes matemáticos. Su aplicabilidad varía desde el uso de pasajes históricos previo al desarrollo de un tema, hasta investigaciones en diversos campos de la matemática, que pretenden cambiar el esquema tradicional de enseñanza.

\section{Conclusiones}

Los programas de estudio para la educación matemática costarricense han presentado diferentes modificaciones desde la promulgación de la Constitución Política de 1949 hasta la reforma educativa de 2012. Estos han encontrado su base en acontecimientos acaecidos en diferentes momentos de la historia política, social y cultural del país.

Los documentos curriculares analizados han planteado cambios desde los contenidos que se deben abarcar en los diferentes niveles del sistema educativo hasta los enfoques de enseñanza y aprendizaje aplicados. La inclusión de la historia de la matemática como componente de la educación matemática responde a la necesidad de cambio en la concepción estudiantil hacia la matemática, y a destacar su origen en la construcción social del conocimiento.

A lo largo del período estudiado, los programas evidencian que la finalidad de incluir elementos históricos en la enseñanza de la matemática apunta hacia el factor motivacional en el discente y su inclusión como parte fundamental del currículo que permite el desarrollo de diferentes situaciones de aprendizaje, es decir, como una herramienta para el profesorado útil en el desarrollo de la clase.

\section{Referencias}

Babini, J. y Pastor, J. (1997). Historia de la matemática. Barcelona, España: Gedisa.

Boyer, C. (2003). Historia de la matemática. Madrid, España: Alianza.

Carrillo, D. (2005). La metodología de la aritmética en los comienzos de las escuelas normales (1838-1868) $y$ sus antecedentes. Murcia, España: Universidad de Murcia.

Castro, L., Cortés, P., Guzmán, R., Lezcano, N., Mora, G. y Rosales, N. (2015). La Historia de la matemática en el currículo de matemáticas de educación secundaria en costa rica (1949-2012) (Tesis de licenciatura). Universidad de Costa Rica, San José, Costa Rica.

Collette, J. P. (1973). Historia de la matemática. México D. F.: Romont. 
UNICIENCIA Vol. 30, No. 2, pp. 1-17. Julio-diciembre, 2016.

ISSN Electrónico: 2215-3470

URL: www.revistas.una.ac.cr/uniciencia

DOI: http://dx.doi.org/10.15359/ru.30-2.1

Email: revistauniciencia@una.cr

Dengo, M. (1995). Educación costarricense (1 $1^{\mathrm{a}}$ ed.). San José, Costa Rica: EUNED.

Dengo, M. (2001). Educación costarricense (1 $1^{\mathrm{a}}$ ed.). San José, Costa Rica: EUNED.

Fauvel, J. y Gray, J. (1991). The History of Mathematics: A reader. Hong Kong, China: Macmillan Press-

The Open Univertity. Fried, M. N. (2001). Can mathematics education and history of mathematics coexist? Science \& Education, 10, 391-408.

Gómez, B. (2000). Los libros de texto de matemáticas. Las matemáticas del siglo XX: Una mirada en 101 artículos. Dialnet, 43, 77-80.

López, C. (2011). La formación de maestros en aritmética y álgebra a través de los libros de texto (Tesis doctoral). Universidad de Salamanca, España. Recuperado de http://gredos.usal.es/jspui/ handle/10366/83280.

Martínez, M. (2012). Cronología de la educación costarricense: Una síntesis histórica - filosófica (1ª ed.). Cartago, Costa Rica: GRAFOS Litografía.

Maz, A. (2005). Los números negativos en España en los siglos XVIII y XIX (Tesis doctoral). Universidad de Granada, España. Recuperado de http://0-hera.ugr.es.adrastea.ugr.es/tesisugr/15378184.pdf

Ministerio de Educación Pública. (1951). Programa de matemáticas. San José, Costa Rica.

Ministerio de Educación Pública. (1964). Programa de matemáticas primer año. San José, Costa Rica: Autor.

Ministerio de Educación Pública. (1964). Programa de matemáticas tercer año. San José, Costa Rica: Autor.

Ministerio de Educación Pública. (1966). Programa de matemáticas primer año. San José, Costa Rica: Autor.

Ministerio de Educación Pública. (1967). Programa de Matemáticas Cuarto Año. San José, Costa Rica: Autor.

Ministerio de Educación Pública. (1967). Programa de matemáticas quinto año. San José, Costa Rica: Autor.

Ministerio de Educación Pública. (1972). Matemática III ciclo. San José, Costa Rica: Autor.

Ministerio de Educación Pública. (1972). Programa de matemáticas correspondiente a cuarto año 1972 y 1979. San José, Costa Rica: Autor.

Ministerio de Educación Pública. (1979). Programa matemáticas educación diversificada undécimo año y optativo. San José, Costa Rica: Autor.

Ministerio de Educación Pública. (1986). Serie Hacia la luz. Matemática 6: Guía para el docente. San José, Costa Rica: Litografía e Imprenta LIL.

Ministerio de Educación Pública. (1987). Programa de estudios: Matemática III y educación diversificada. San José, Costa Rica: Autor.

Ministerio de Educación Pública. (1995). Programa de estudios: Matemática III ciclo. San José, Costa Rica: Autor.

Ministerio de Educación Pública. (1996). Programa matemáticas educación diversificada. San José, Costa Rica: Autor.

Ministerio de Educación Pública. (2000). Programa de estudios: Matemática III ciclo. San José, Costa Rica: Autor.

Ministerio de Educación Pública. (2005). Programa de estudios: Matemática educación diversificada. San José, Costa Rica: Autor.

Ministerio de Educación Pública. (2005). Programa de estudios: Matemática III ciclo. San José, Costa Rica: Autor. 
ISSN Electrónico: 2215-3470

DOI: http://dx.doi.org/10.15359/ru.30-2.1
UNICIENCIA Vol. 30, No. 2, pp. 1-17. Julio-diciembre, 2016.

URL: www.revistas.una.ac.cr/uniciencia Email: revistauniciencia@una.cr

Ministerio de Educación Pública. (2012). Reforma curricular en ética, estética y ciudadanía. Programas de estudio de matemáticas, I, II y III ciclos de la educación general básica y ciclo diversificado. San José, Costa Rica: Autor.

Ministerio de Educación Pública. (2012). Programas de estudio matemáticas. I, II y III ciclos de la educación general básica y ciclo diversificado. San José, Costa Rica: Autor.

Picado, M. (2012). El sistema métrico decimal en libros de texto de matemáticas en España durante la segunda mitad del siglo XIX (1849-1892) (Tesis doctoral). Universidad de Granada, España. Recuperado de http://fqm193.ugr.es/produccion-cientifica/tesis/ver_detalles/7464/

Quesada, R. (2003). Estado y educación en Costa Rica: Del agotamiento del liberalismo al inicio del Estado interventor 1914-1949. San José, Costa Rica: Editorial de la Universidad de Costa Rica.

Rama, G. W. (1994). A la búsqueda del siglo XXI: Nuevos caminos de desarrollo en Costa Rica. San José, Costa Rica: Editorial de la Universidad de Costa Rica.

Retana, C. (2010). Currículo de la educación media costarricense de 1950 a 2010. San José, Costa Rica: CONARE.

Ruíz, A. y Barrantes, H. (2000). La reforma liberal y las matemáticas en la Costa Rica del siglo XIX. Llull: Revista de la Sociedad Española de Historia de las Ciencias y de las Técnicas, 23(46), 145-172.

Sierra, M. (1997). Notas de historia de la matemática para el currículo de secundaria. En L. Rico (Coord.), La educación matemática en la enseñanza secundaria (pp. 179-194). Barcelona, España: Horsori.

UNESCO. (1990). Declaración Universal de la Educación para Todos. Jomtien, Tailandia.

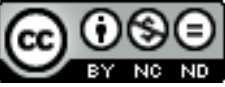

La historia como recurso didáctico para la enseñanza de las matemáticas desde las directrices curriculares para la Educación Secundaria en Costa Rica (1949-2012) (Leonel Castro-Soto y otros) por Revista Uniciencia se encuentra bajo una Licencia 